
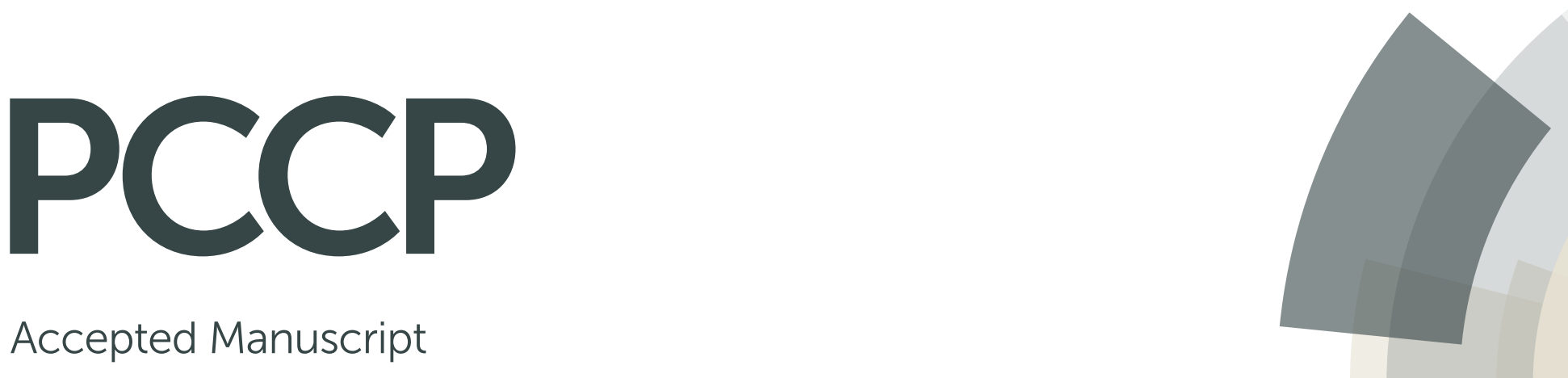

Accepted Manuscript

This article can be cited before page numbers have been issued, to do this please use: A. Latimer, $H$. Aljama, A. Kakekhani, J. S. Yoo, A. Kulkarni, C. Tsai, M. Garcia-Melchor, F. Abild-Pedersen and J. K. Nørskov, Phys. Chem. Chem. Phys., 2017, DOI: 10.1039/C6CP08003K.

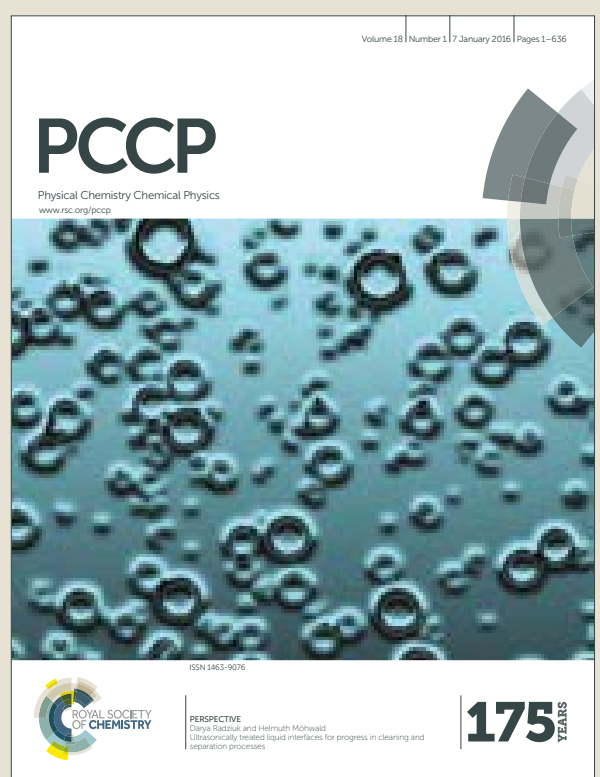

This is an Accepted Manuscript, which has been through the Royal Society of Chemistry peer review process and has been accepted for publication.

Accepted Manuscripts are published online shortly after acceptance, before technical editing, formatting and proof reading. Using this free service, authors can make their results available to the community, in citable form, before we publish the edited article. We will replace this Accepted Manuscript with the edited and formatted Advance Article as soon as it is available.

You can find more information about Accepted Manuscripts in the author guidelines.

Please note that technical editing may introduce minor changes to the text and/or graphics, which may alter content. The journal's standard Terms \& Conditions and the ethical guidelines, outlined in our author and reviewer resource centre, still apply. In no event shall the Royal Society of Chemistry be held responsible for any errors or omissions in this Accepted Manuscript or any consequences arising from the use of any information it contains. 


\section{Mechanistic Insights into Heterogeneous Methane Activation}

Allegra A. Latimer, ${ }^{1}$ Hassan Aljama, ${ }^{1}$ Arvin Kakekhani, ${ }^{1}$ Jong Suk Yoo, ${ }^{1}$ Ambarish Kulkarni, ${ }^{1}$ Charlie Tsai, ${ }^{1}$ Max GarciaMelchor, ${ }^{1}$ Frank Abild-Pedersen, ${ }^{1,2}$ Jens K. Nørskov ${ }^{1,2, *}$

${ }^{1}$ SUNCAT Center for Interface Science and Catalysis, Department of Chemical Engineering, Stanford University, 450 Serra Mall Stanford, California 94305, USA

${ }^{2}$ SUNCAT Center for Interface Science and Catalysis, SLAC National Accelerator Laboratory, 2575 Sand Hill Road, Menlo Park, California 94025, United States

* corresponding author: norskov@stanford.edu 
While natural gas is an abundant chemical fuel, its low volumetric energy density has prompted a search for catalysts able to transform methane into more useful chemicals. This search has often been aided through the use of transition state (TS) scaling relationships, which estimate methane activation TS energies as a linear function of a more easily calculated descriptor, such as final state energy, thus avoiding tedious transition state energy calculations. It has been shown that methane can be activated via a radical or surfacestabilized pathway, both of which possess a unique TS scaling relationship. Herein, we present a simple model to aid in the prediction of methane activation barriers on heterogeneous catalysts. Analogous to the universal radical TS scaling relationship introduced in a previous publication, we show that a universal TS scaling relationship that transcends catalysts classes also seems to exist for surface-stabilized methane activation if the relevant final state energy is used. We demonstrate that this scaling relationship holds for several reducible and irreducible oxides, promoted metals, and sulfides. By combining the universal scaling relationships for both radical and surface-stabilized methane activation pathways, we show that catalyst reactivity must be considered in addition to catalyst geometry to obtain an accurate estimation for the TS energy. This model can yield fast and accurate predictions of methane activation barriers on a wide range of catalysts, thus accelerating the discovery of more active catalysts for methane conversion.

It is estimated that approximately $200 \times 10^{12}$ cubic meters of methane remain uncaptured in the earth's crust ${ }^{1}$. If methane hydrates are included, the number could be as high as $15 \times 10^{15}$ cubic meters ${ }^{1}$. Unfortunately, while abundant, methane is not an ideal source of chemical energy. Its low volumetric energy density often renders transportation from remote locations uneconomical and prevents it from being used widely as a transportation fuel ${ }^{2}$. For these reasons, a vast body of research has focused on transforming methane into more energy dense fuels or higher value commodity chemicals ${ }^{3-8}$. Irrespective of final product, these transformations all begin with methane activation - namely, the scission of one of the four carbon-hydrogen bonds. Therefore, a thorough understanding of this initial step has the ability to inform research across the diverse field of methane oxidation.

It has been shown previously that methane activation occurs via one of two possible pathways $^{9}$ : in the first, the methyl group is stabilized by the catalyst, while, in the second, a radicallike intermediate is formed in which the methyl group is stabilized by the $\mathrm{OH}$ bond rather than the 
surface $^{10}$. These two pathways lead to distinctly different transition state (TS) energy scaling behavior. TS scaling relationships generally describe the linear dependence of TS energies on more easily calculated descriptors, such as the energy of the final state or that of a key adsorbate. These relationships facilitate first-pass catalyst screening by allowing a TS energy to be estimated before performing a resource-intensive barrier calculation.

The methane activation TS energies of systems that proceed through a radical-like pathway scale with the hydrogen abstraction energy of the active site, $\mathrm{E}_{\mathrm{H}}{ }^{11,12}$, while the TS energies of metals and transition metal compounds that proceed through the surface-stabilized pathway scale with the final state energy, $\mathrm{E}_{\mathrm{FS}}{ }^{13,14}$. Therefore, it is important to understand which catalyst properties favor one pathway over the other so that the proper scaling relation may be used to estimate the TS energy. Clearly, in catalysts with isolated active sites such as zeolites and other porous materials, the radical pathway will be favored due to the large distance between active sites. Similarly, as a surface-stabilized pathway intuitively seems more energetically favorable than the formation of a methyl radical, it has previously been assumed that catalysts with a high density of active sites, such as metals, will activate methane via methyl-surface stabilization ${ }^{15,16}$. Surprisingly, however, our findings suggest that this is not necessarily the case. Herein, we demonstrate that reactivity must be considered in addition to catalyst structure to determine the lowest energy methane activation pathway. We show that catalysts that possess the appropriate geometry to activate methane via surface stabilization will prefer the radical pathway if the methyl binding energy is sufficiently low. To address this, we find that both the universal radical ${ }^{12}$ and a surface-stabilized TS scaling relationship can be used to estimate two TS energies. The scaling relationship that gives the lower TS energy will correspond to the favored pathway, facilitating the prediction of methane activation barriers on a wide range of catalysts.

To explore how the methane activation pathway depends on catalyst reactivity, we choose FCC (111) metals as a model system due to their prevalence in catalysis and their established ability to activate methane via the surface-stabilized pathway ${ }^{13,15}$. We examined oxygen promoted and clean surfaces, as each is relevant to methane activation under different conditions and on different metals ${ }^{15}$. To determine what catalyst electronic properties favor one pathway over the other, we calculated both the surface-stabilized and radical pathways on all surfaces. By setting the final state of the system to be either $(\mathrm{O}) \mathrm{H}^{*}+\mathrm{CH}_{3}(\mathrm{~g})$ or $(\mathrm{O}) \mathrm{H}^{*}+\mathrm{CH}_{3} *$ (where there is an oxygen in the case of oxygen-promoted metals), we were able to isolate radical TSs even when the surface- 
stabilized pathway was preferred. Initial, transition and final state example geometries are shown in Fig. 1. On non-promoted (clean) metal surfaces, no saddle point was found between the initial state, $\mathrm{CH}_{4}(\mathrm{~g})$, and the radical final state, $\mathrm{CH}_{3}(\mathrm{~g})+\mathrm{H}^{*}$. Therefore, for these cases, we assume the radical TS is very final-state-like and approximate its energy to be that of the methyl radical, $\mathrm{CH}_{3}(\mathrm{~g})+\mathrm{H}^{*}$. A comprehensive list of all radical and surface-stabilized TS energies considered in this work are listed in Table S1.

IS

(a)

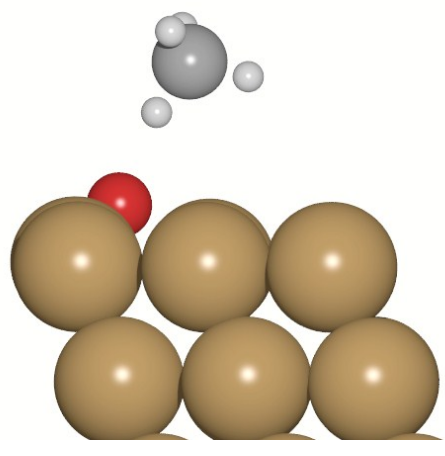

(b)

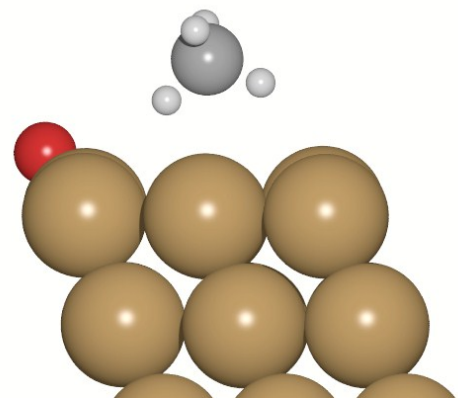

TS
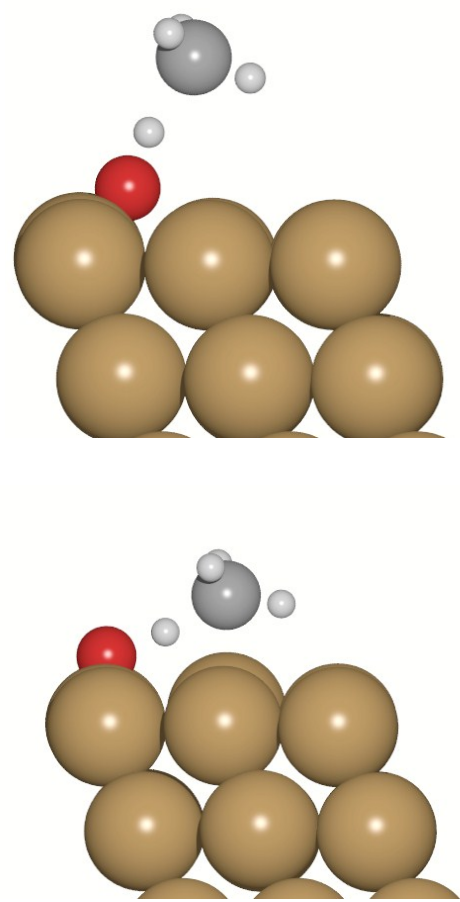

FS
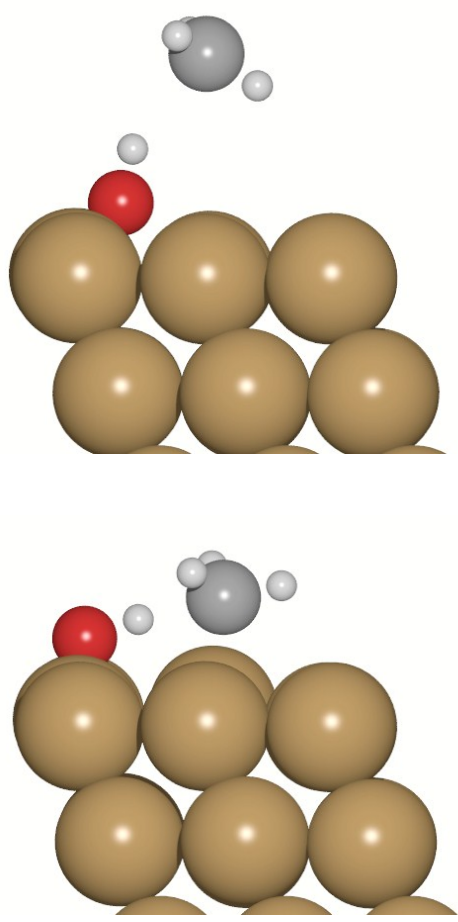

Figure 1. Example geometries of initial state (IS), transition state (TS) and final state (FS) for (a) radical and (b) surface-stabilized pathways

Surprisingly, we find that the oxygen-promoted noble metals, Au and Ag, proceed through the radical-like TS regardless of the starting geometry for the calculation. Because a surfacestabilized TS could not be isolated for these materials, it can be assumed that their preferred TS is radical-like. This finding is significant because both $\mathrm{Au}$ and $\mathrm{Ag}$ have the appropriate geometry to proceed through the surface-stabilized pathway, implying that the preference of these noble metals 
to proceed through a radical-like pathway must be due to their electronic structures. While there is likely also a structural dependence if active centers for $\mathrm{H}$ stabilization and $\mathrm{CH}_{3}$ stabilization are far apart, this effect does not seem to be dominant on metal surfaces. Therefore, the preference of a catalyst to proceed through one pathway or the other will be governed by the relative energies of the corresponding TSs. This conclusion can be visualized by plotting TS energies from both pathways as a function of $\mathrm{E}_{\mathrm{H}}$. $\mathrm{E}_{\mathrm{H}}$ is defined analogously to a previous publication ${ }^{12}$ as:

$E_{H}=E\left(M_{m} O_{x} H_{y+1}\right)-E\left(M_{m} O_{x} H_{y}\right)$

Where $E\left(M_{m} O_{x} H_{y+1}\right)$ and $E\left(M_{m} O_{x} H_{y}\right)$ are the formation energies of the hydrogenated and dehydrogenated active sites, respectively, referenced to gas-phase $\mathrm{H}_{2} \mathrm{O}$ and $\mathrm{O}_{2}$. As previously discussed, radical-like methane activation TS energies can be completely described by $\mathrm{E}_{\mathrm{H}}$ according to the linear scaling relationship in Eq. 2. Alternatively, surface-stabilized TS energies are a linear function of $\mathrm{E}_{\mathrm{FS}}$, which can be written as $E_{C H 3}+E_{H}$ in systems without strong adsorbate-adsorbate interactions (Eq. 3) ${ }^{13}$. If only metals are considered, surface-stabilized TS energies can also be projected directly onto $\mathrm{E}_{\mathrm{H}}$ due to the linear scaling that exists between $\mathrm{E}_{\mathrm{CH} 3}$ and $\mathrm{E}_{\mathrm{H}}$ on metals (Fig. 2, Eq. 4-6) ${ }^{14}$, where $\gamma$ and $\delta$ will be different for O-promoted or clean surfaces.

$$
\begin{aligned}
& E_{T S}=0.75 E_{H}+1.09 \\
& E_{T S}=0.67\left(E_{C H 3}+E_{H}\right)+1.04 \\
& E_{C H 3}=\gamma E_{H}+\delta \\
& E_{T S}=0.67\left(\gamma E_{H}+\delta+E_{H}\right)+1.04 \\
& E_{T S}=0.67(1+\gamma) E_{H}+0.67 \delta+1.04
\end{aligned}
$$




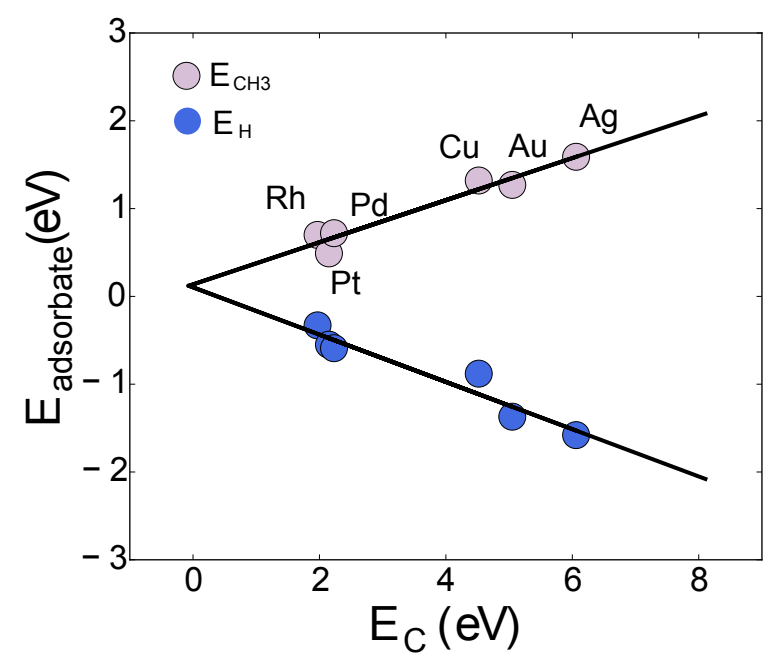

Figure 2. Scaling of $\mathrm{E}_{\mathrm{CH} 3}$ and $\mathrm{E}_{\mathrm{H}}$ as a function of $\mathrm{E}_{\mathrm{C}}$ on O-Promoted FCC (111) metals

Fig. 3 shows the TS energies calculated for both radical and surface-stabilized pathways on FCC (111) metals as a function of $\mathrm{E}_{\mathrm{H}}$. As expected, all radical TSs (triangles) follow the universal radical TS scaling line (black). Where the universal radical TS scaling line intersects the surface-stabilized TS scaling lines for oxygen-promoted (red) or clean (blue) metals, catalysts less reactive than the intersection point begin to favor the radical pathway as it now has the lower of the two TS energies. The change in TS geometry for oxygen-promoted Au and Ag can therefore be fully attributed to the radical pathway having a lower TS energy than the surface-stabilized pathway on these surfaces. The intersection of the radical and surface-stabilized scaling lines for a given class of materials determines where a "mechanistic switch" will occur. 


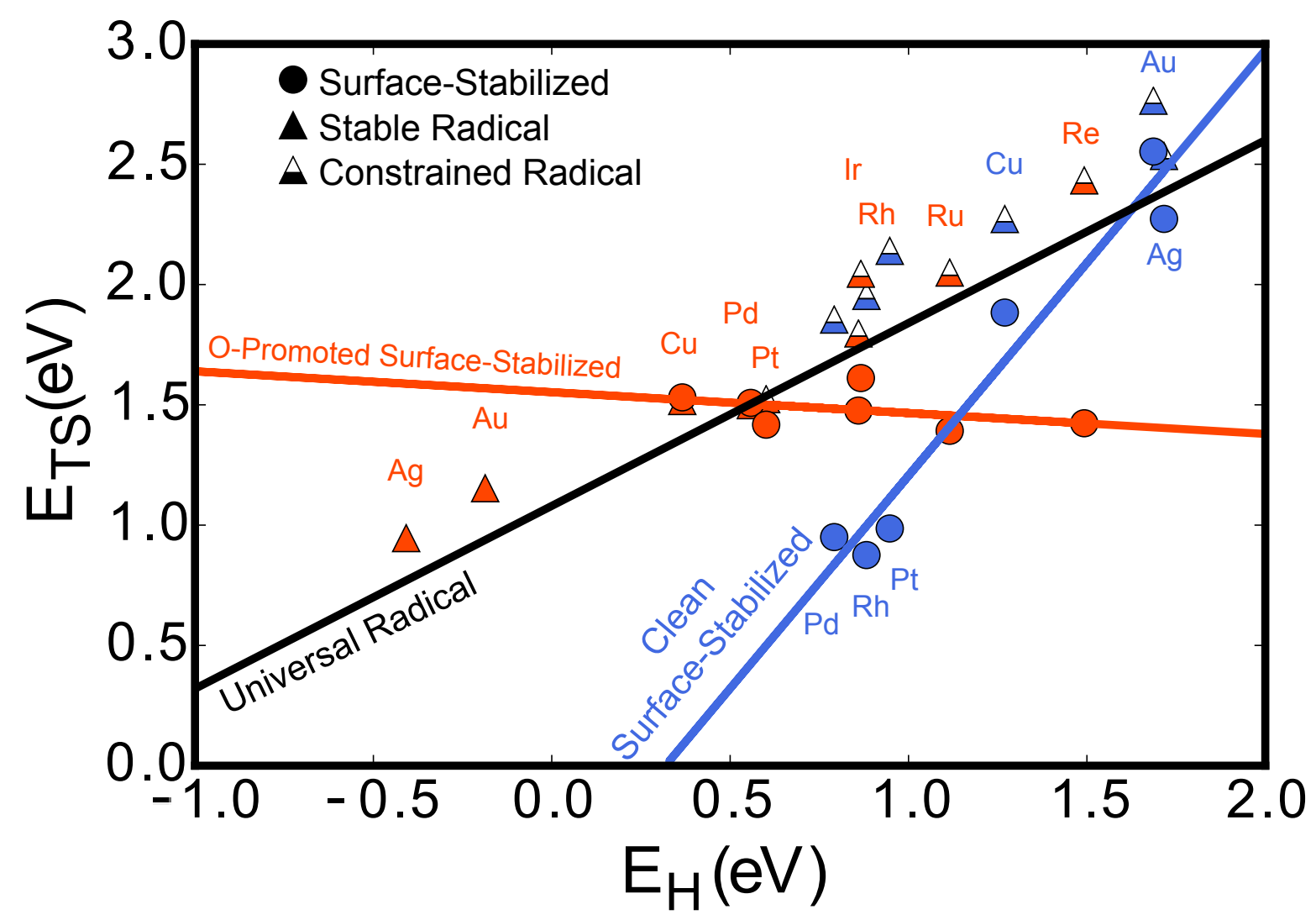

Figure 3. Comparison of radical (triangles) and surface-stabilized (circles) methane activation on Opromoted (red; $\mathrm{y}=-0.07 \mathrm{x}+1.53$, MAE=0.04) and clean (blue; $\mathrm{y}=1.77 \mathrm{x}-0.56, \mathrm{MAE}=0.15)$ FCC (111) metal surfaces. The half-filled shapes correspond to non-energetically preferred TS geometries, while the filled shapes correspond to stable TS geometries.

A noteworthy feature of Fig. 3 is the mirroring effect that arises when comparing oxygenpromoted to clean metals. Namely, the oxygen-promoted noble catalysts have the most favorable $\mathrm{E}_{\mathrm{H}}$, while the opposite is true for clean noble metals. This observation can be explained by considering the effect of a promoting species on a catalyst. A promoter on a reactive catalyst will be bound strongly and be relatively unreactive, while a promoter on a noble catalyst will be bound very weakly to the surface and thus be more reactive ${ }^{14,15}$. Therefore, the hydrogen abstraction energy of a clean gold surface will be unfavorable, whereas that of a weakly-bound, reactive oxygen atom adsorbed on gold will be favorable. This idea points to another aspect of the plot that may be surprising to some researchers: the barriers for oxygen-promoted methane activation on the noble metals, gold and silver, are quite low, having nearly the same energy as the non- 
promoted reactive metals, such as Pt and Pd. Given the dearth of successful examples in which noble metal catalysts convert methane to useful products in the literature, such a finding may seem unexpected $^{17}$. However, this discrepancy can likely be attributed to the low oxygen coverages that exist at most relevant oxygen chemical potentials on noble metals due to their unfavorable formation energies ${ }^{18}$. It should also be noted that a more stable radical TS does not imply the final state will include a methyl radical; even on $\mathrm{Ag}$ and $\mathrm{Au}, \mathrm{CH}_{3} *$ is approximately $0.8 \mathrm{eV}$ more stable than a gas-phase radical.

The near-zero slope of the surface-stabilized O-promoted metals can be explained by a bond-order conservation argument. As mentioned previously, the TS energy scales with $\mathrm{E}_{\mathrm{CH}_{3}}+\mathrm{E}_{\mathrm{H}}$. $\mathrm{E}_{\mathrm{CH} 3}$ scales has a slope of $1 / 4$ when plotted against $\mathrm{E}_{\mathrm{C}}$, since three of four possible bonds are already saturated (Fig. 2) ${ }^{19}$. $\mathrm{E}_{\mathrm{H}}$, on the other hand, scales with $\mathrm{E}_{\mathrm{C}}$ with a slope of -1/4, the origins for which is discussed in greater detail in the SI (Fig. 2) ${ }^{14}$. Therefore, $\mathrm{E}_{\mathrm{CH} 3}+\mathrm{E}_{\mathrm{H}}$ is effectivity constant for Opromoted metals, leading to a case where the TS energy varies little as a function of $E_{H}$.

While the idea that a methyl radical TS would be more stable than a surface-stabilized TS initially seems unintuitive, Fig. 3 remarkably shows a region where the interaction of the methyl group with the catalyst surface destabilizes the TS. In this region, noble metals drop to the universal radical scaling line and energetically favor the radical pathway. It is worth noting that the surface-stabilized TS scaling relation estimates the TS to be on the order of $1 \mathrm{eV}$ higher than the true, radical-like TS. Therefore, surprisingly, it is not sufficient to assume methane activation will occur via the surface-stabilized pathway, even if the catalyst has the appropriate structure to do so.

Unfortunately, many other interesting catalyst materials, such as oxides, do not exhibit reliable scaling between $\mathrm{E}_{\mathrm{CH} 3}$ and $\mathrm{E}_{\mathrm{H}}$ and therefore cannot be visualized in the same way as the metals shown in Fig. 3. However, these materials do follow the universal radical scaling ${ }^{12}$ (Fig. 4a) if methane is activated via the radical-like pathway. Additionally, all materials we explored that proceed through the surface-stabilized pathway, including promoted metals, sulfides, and oxides, seem to follow the previously-established TS scaling relationship for $\mathrm{CH}_{\mathrm{x}}$ activation on 111 and 211 metals as shown in Fig. $4 b^{13,14}$. The apparent universality of surface-stabilized TS scaling implies that these two "universal" scaling relationships can now be used to describe heterogeneous methane activation nearly comprehensively. In other words, for any system with the appropriate 
geometry to activate $\mathrm{CH}_{4}$ via a surface-stabilized pathway ${ }^{\mathrm{a}}$, the preference for a radical TS can be tested using these two scaling relationships. If a TS energy is calculated for both pathways using the corresponding scaling relationships, the lower of the two energies will be:

$E_{T S}=\operatorname{minimum}\left(f_{\text {radical }}\left(E_{H}\right), f_{\text {surface-stabilized }}\left(E_{F S}\right)\right)$

Here, $f_{\text {radical }}\left(E_{H}\right)$ is the universal radical TS scaling ${ }^{12}$ (Eq. 2) and $f_{\text {surface-stabilized }}\left(E_{F S}\right)$ is the universal surface-stabilized TS scaling ${ }^{13}$ (Eq. 3). Because all materials that lack the appropriate geometry for surface-stabilized $\mathrm{CH}_{4}$ activation will automatically be described by the universal radical scaling line ${ }^{12}$, this approach facilitates the prediction of methane activation barriers on nearly any catalyst.
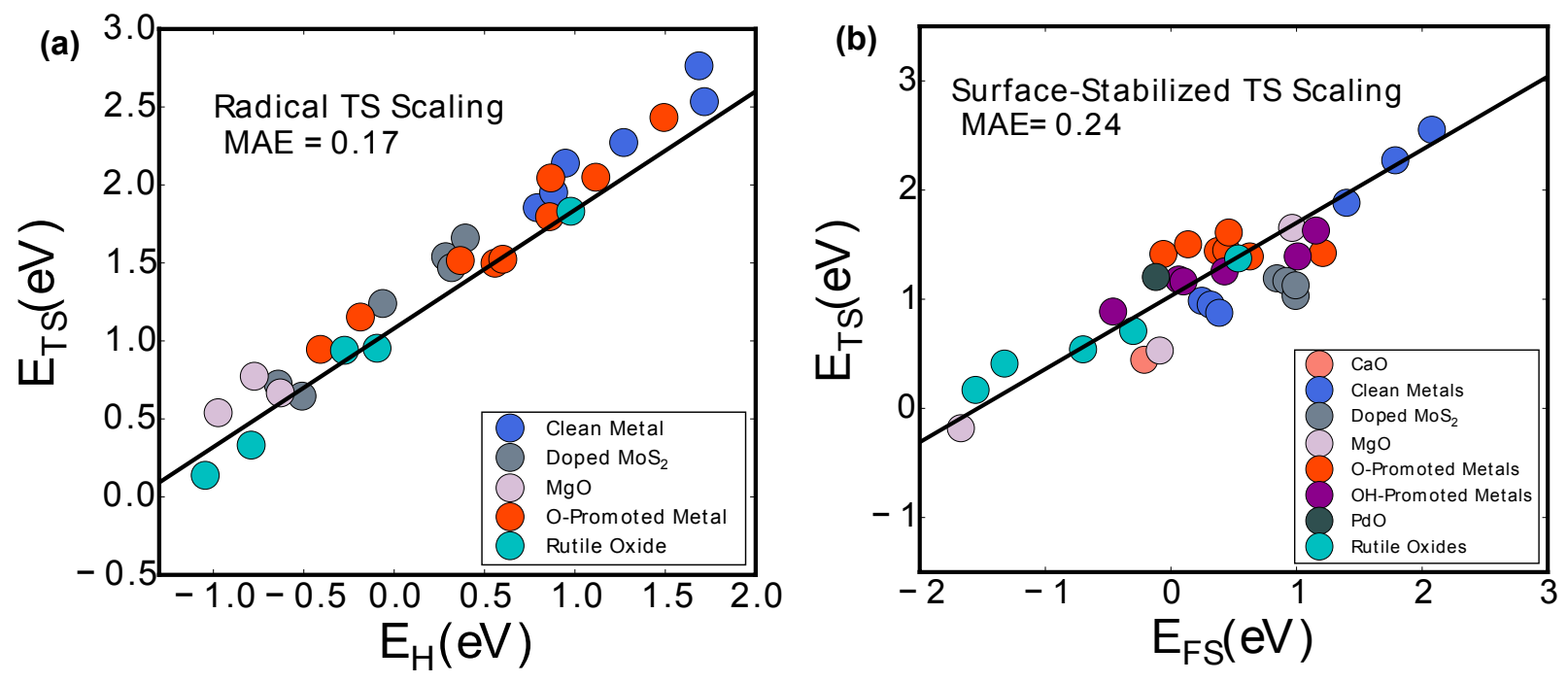

Figure 4. Universal scaling relations for (a) the radical pathway $\left(0.75 \mathrm{E}_{\mathrm{H}}+1.09\right)$ and (b) the surfacestabilized pathway $\left(0.67 \mathrm{E}_{\mathrm{FS}}+1.04\right)$.

\footnotetext{
${ }^{\text {a }}$ It should be stressed that the model presented in Eq. 6 should only be used for systems where surface-stabilized TSs are geometrically accessible. A catalyst with distant active sites, for example, might energetically favor a surface-stabilized TS, but if its structure prevents it from accessing this pathway it will proceed through the radical pathway.
} 
There are several noteworthy aspects of Fig. 4b. First, it should be clarified that the FS energies of insulating systems include adsorbate-adsorbate interactions. In other words, the independent variable for these points is $\mathrm{E}_{\mathrm{H}+\mathrm{CH} 3}$ rather than $\mathrm{E}_{\mathrm{H}}+\mathrm{E}_{\mathrm{CH} 3}$. The reason for this distinction lies in the localized nature of adsorption on insulators. In these systems, intermediates' adsorption energies are significantly altered in the presence of other adsorbed species ${ }^{20}$. Adsorbate-adsorbate interactions will also to a lesser degree affect the scaling of the other catalyst classes in Fig. 4b, but these deviations are not regarded as significant for metallic systems. Fig. 4b also exhibits minimal variation in both the domain and range of O-promoted metals. This observation can be rationalized by considering the equal and opposite scaling of $\mathrm{E}_{\mathrm{H}}$ and $\mathrm{E}_{\mathrm{CH} 3}$ in O-promoted metals, as discussed earlier (Fig. 2). Because $\mathrm{E}_{\mathrm{CH} 3}$ will exactly compensate any changes in $\mathrm{E}_{\mathrm{H}}$ and vice versa, the independent variable, $\mathrm{E}_{\mathrm{FS}}$, is effectively constant for all surfaces. A final note on Fig. $4 \mathrm{~b}$ relates to the catalyst whose TS energy is seemingly less than zero. This effect can be understood by recognizing that several of the oxides studied herein bind methane relatively strongly $(\sim 0.5 \mathrm{eV})$, so that if the methane dissociation barrier on these oxides is sufficiently low, the TS energy will be negative when referenced to gas-phase methane. If the reference were instead $\mathrm{CH}_{4}{ }^{*}$, small but positive TS energies would be observed.

The model given by Eq. 7 can be visualized in a 2D plot as shown in Fig. 5a, where the two independent descriptors, $\mathrm{E}_{\mathrm{H}}$ and $\mathrm{E}_{\mathrm{FS}}$, fully describe the TS energy. The black line marks $\mathrm{E}_{\mathrm{H}}$ and $E_{F}$ values where the "mechanistic switch" will occur; points to its left will be radically activated, and points to its right will be surface-stabilized. Fig. 5b shows the accuracy of using Eq. 7 to predict TS energies compared to the corresponding DFT transition state energies. Notably, only two energies, $\mathrm{E}_{\mathrm{FS}}$ and $\mathrm{E}_{\mathrm{H}}$, are needed to predict TS energies for many catalysts with an $\mathrm{MAE}=0.24 \mathrm{eV}$. The success of this model in describing metals, promoted metals, metallic and insulating oxides, and sulfides suggests that it can be used with confidence to assess the reactivity of many new potential methane activation catalysts. Using this model as a first-pass assessment will allow the user to determine the most likely TS geometry and energy for methane activation. This knowledge can be used to optimize initial guesses in a full barrier calculation or stand alone as an accurate estimation of the TS energy on nearly any catalyst. 

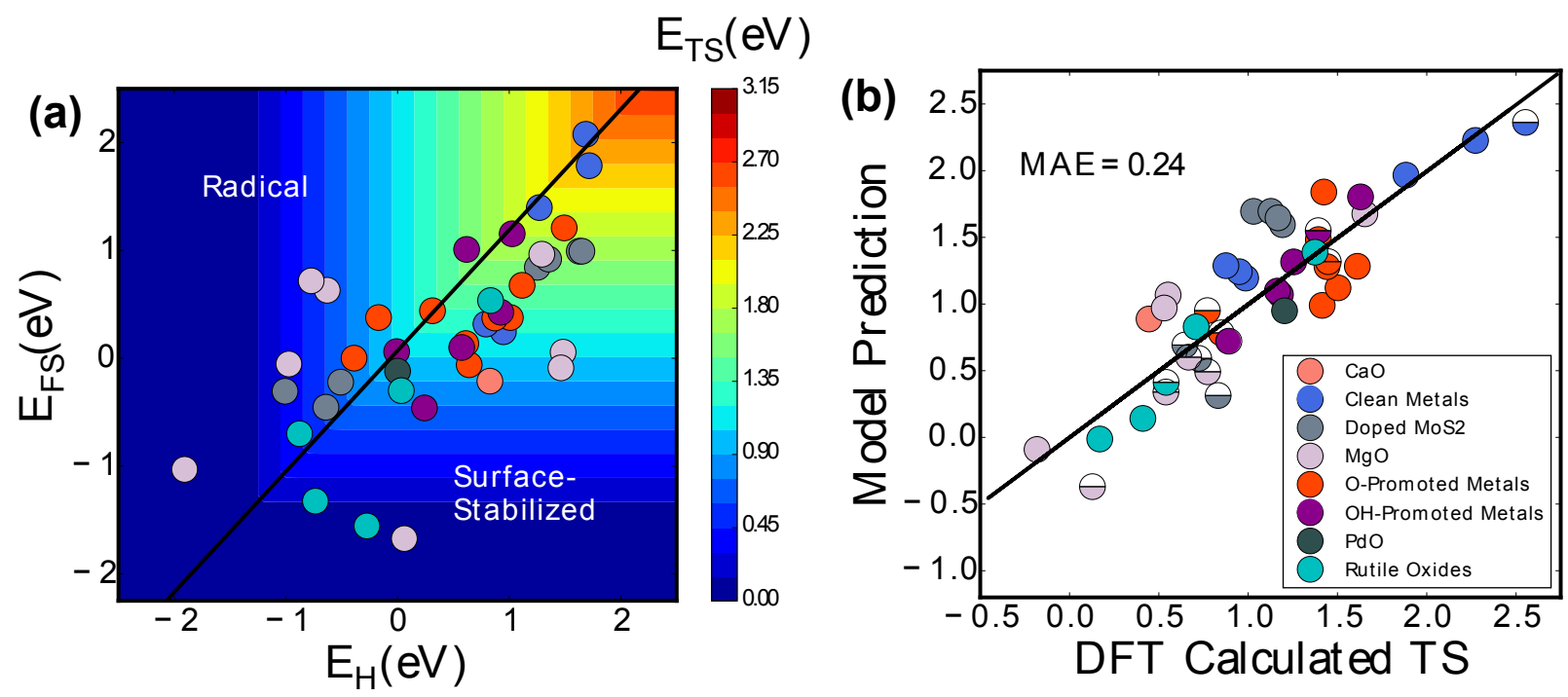

Figure 5. Model for predicting methane activation TS energies as given by Eq. 7. (A) $E_{T S}$ as a function of two independent descriptors, $\mathrm{E}_{\mathrm{FS}}$ and $\mathrm{E}_{\mathrm{H}}$. The equation of the black line is $\mathrm{E}_{\mathrm{FS}}=1.12 \mathrm{E}_{\mathrm{H}}+0.07$, which marks the points where the two scaling relationships predict equal TS energies. (B) Accuracy of model prediction compared to DFT-calculated TSs (MAE=0.24, maximum absolute error: $0.41 \mathrm{eV}$ ). Full circles are surfacestabilized transition states, and half-filled circles are radical transition states.

To determine the applicability of this model to catalysts with less traditional geometries than examined thus far, we decided to explore methane activation on the catalyst shown in Fig. 6a, which we refer to as $\mathrm{O} @ \mathrm{Ca} @ \mathrm{O} @ \mathrm{MoS}_{2}$. On a $\mathrm{MoS}_{2}$ slab with sulfur vacancies, exposure to oxygen would fill these vacancies resulting in $\mathrm{O} @ \mathrm{MoS}_{2}$. The slight electronegativity difference between oxygen and sulfur would lead any calcium ions doped into the system to migrate to oxygen sites $\left(\mathrm{Ca} @ \mathrm{O} @ \mathrm{MoS}_{2}\right)$, and further exposure to oxygen would lead to oxygen adsorption on the calcium ion resulting in the final active site. A surface-stabilized TS was isolated (Fig. 6b), in which the methyl is stabilized on the same calcium to which to H-abstracting oxygen is bound. This unusual TS geometry followed the SS scaling line well, as shown in Fig. 6d. However, when provided $\mathrm{E}_{\mathrm{H}}$ and $\mathrm{E}_{\mathrm{FS}}$ for this active site, the model suggested a more energetically favorable radical-like TS existed. Indeed, this radical-like TS was isolated and found to have a lower energy (Fig. 6c). The success of the model in describing methane activation on this unusual active site demonstrates its potential to accurately describe a wide range of catalyst materials. 

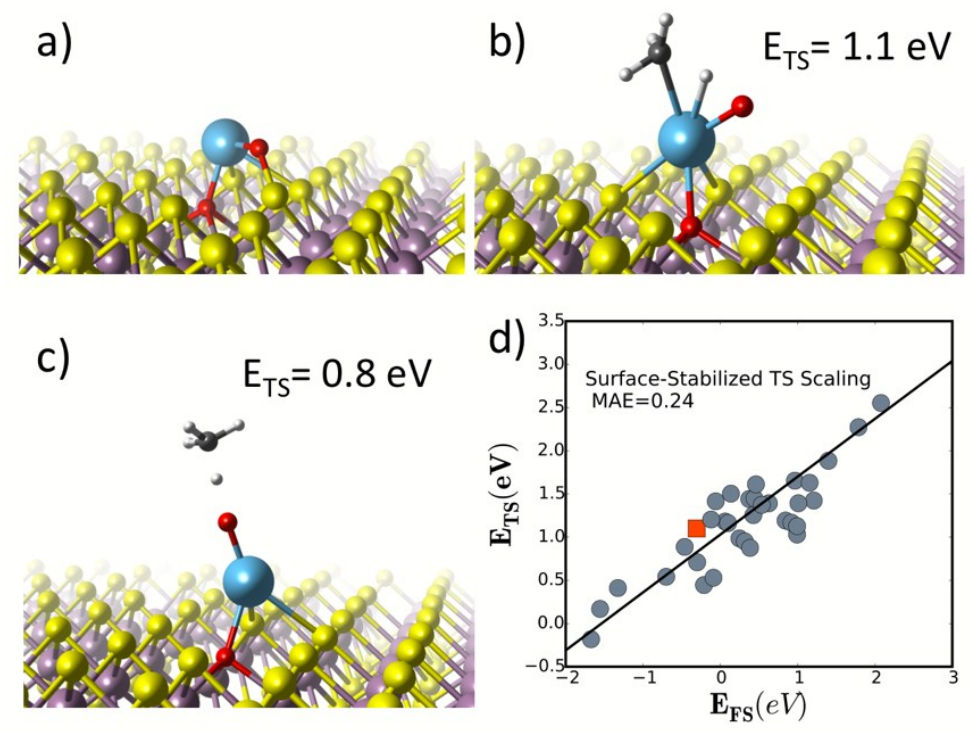

Figure 6. The model extended to a less traditional active site geometry, $\mathrm{O} @ \mathrm{Ca} @ \mathrm{O} @ \mathrm{MoS}_{2}$. Here, (a) is the active site before methane activation, (b) is the TS for the SS pathway, (c) is the TS for the radical-like pathway, and (d) shows the good agreement of the SS TS with its corresponding scaling relationship. In all subfigures, atom colors are as follows: S: yellow; Mo: purple; Ca: blue; O: red; C: grey; H: red.

Previously, we noted that the model described herein should only be applied to catalysts in which the surface-stabilized pathway is geometrically accessible. This is a logical conclusion when considering the extreme example of porous materials, such as zeolites, with distances between active sites on the order of tens or hundreds of angstroms. Regardless of how energetically favorable the $\mathrm{CH}_{3}$ binding energy might be, sites that are too far apart will not be able to aid in stabilization of the TS. However, this analysis prompts the following question: at what distance between active sites does the surface-stabilization become possible? Herein, we have underscored previously-established data ${ }^{15}$ showing that a distance of $\sim 2 \AA$ between $\mathrm{H}^{*}$ and $\mathrm{CH}_{3} *$ in the final state of methane activation on clean FCC (111) metals is small enough for surface stabilization to take place. However, we find that stabilization does not occur on oxygen-covered rutile oxides, where $\mathrm{H}^{*}$ and $\mathrm{CH}_{3} *$ in the FS are separated by $\sim 3 \AA$, even in cases where $\mathrm{CH}_{3}$ binding is very favorable (Fig. S1). This suggests, as one might expect, that there is a tradeoff between stabilization energy gained by the methyl group interacting with the surface and the unfavorable energy of stretching the $\mathrm{C}-\mathrm{H}$ bond too far in the TS. Interestingly, this distance dependence is not seen on active sites where an acid-base pair is present. For example, methane is activated via the 
surface-stabilized pathway over an $\mathrm{O}^{\mathrm{b}}$ and $\mathrm{a}^{\mathrm{c}}$ on 110 oxide surfaces, even though the distance between these active sites is again $\sim 3 \AA$ (Fig. S2). This may be rationalized by arguing that the heterolytic bond cleavage favored by acid-base pairs will be governed by more long-range ionic interactions than the homolytic cleavage that occurs when no acid-base pair is present to polarize the bond ${ }^{21}$. Hence, in order to highlight situations where caution should be taken when predicting pathways and barriers for methane activation, we present a simple flowchart (Fig. 7).

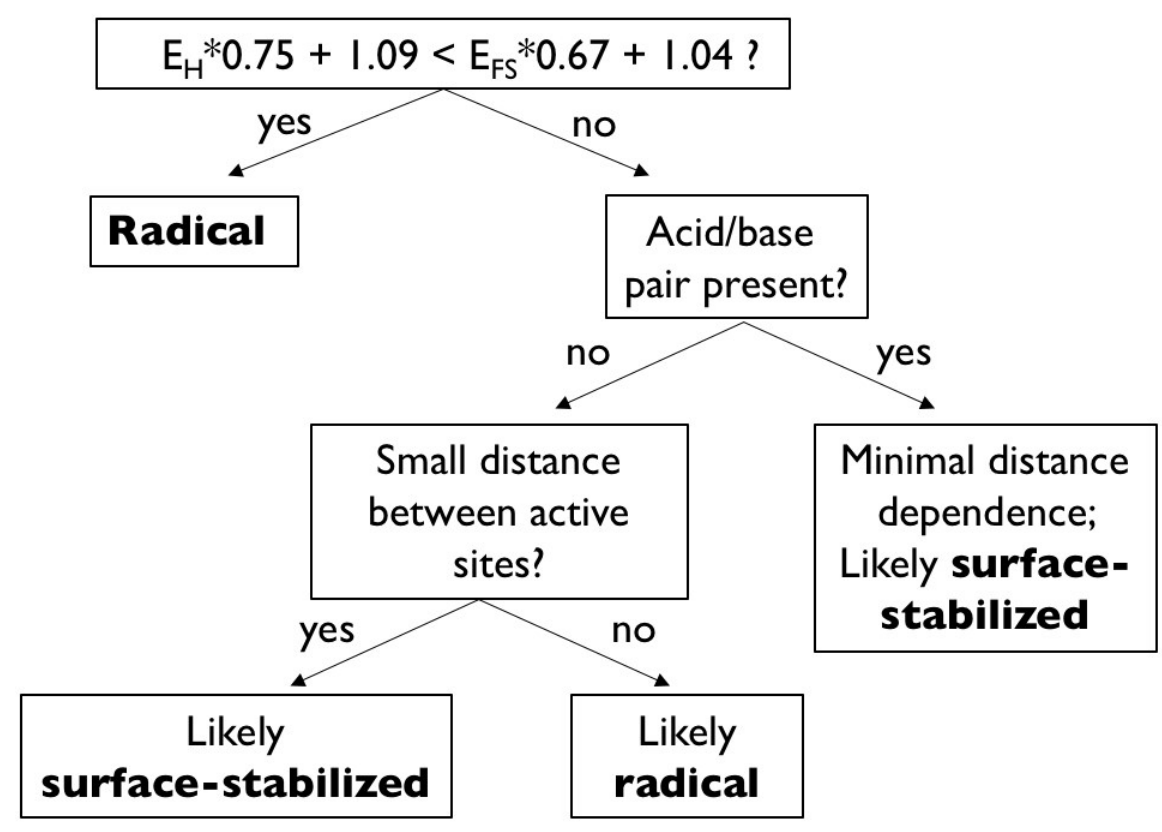

Figure 7. Flowchart outlining different possible methane activation pathways.

While the distance dependence of homolytic C-H bond cleavage has not yet been quantified, the model presented herein can nevertheless be applied with confidence to active sites that fall in the predictable regions of the flowchart or those with structures that have previously been shown to activate methane via the surface-stabilized pathway.

We have demonstrated the existence of a second universal TS scaling relationship for the surface-stabilized methane activation pathway. By combining this with the previously described universal radical TS scaling ${ }^{12}$, we formulated a simple model to predict which pathway will be preferred to activate methane on a given catalyst site, facilitating the subsequent use of the correct scaling relationship. This model elucidates the need to consider catalyst electronic structure in 
addition to catalyst geometry when determining the pathway for methane activation, and can be used to quickly estimate methane activation barriers on a number of catalysts.

\section{Acknowledgements}

Support from the U.S. Department of Energy Office of Basic Energy Science to the SUNCAT Center for Interface Science and Catalysis is gratefully acknowledged. The research of AAL was conducted with Government support under and awarded by DoD, Air Force Office of Scientific Research, National Defense Science and Engineering Graduate (NDSEG) Fellowship, 32 CFR 168a. CT acknowledges support from the National Science Foundation Graduate Research Fellowship Program (GRFP) Grant DGE-114747. JSY appreciates the financial support from US DOS via International Fulbright Science \& Technology Award program. HA receives funding from Aramco Services Company.

\section{Methods:}

The plane wave QuantumEspresso code and Bayesian Error Estimation Functional with Van der Waals corrections (BEEF-vdw) functional was used for the Density Functional Theory (DFT) calculations. The plane-wave cutoff and density cutoff were $500 \mathrm{eV}$ and $5000 \mathrm{eV}$, respectively. Forces on all atoms were minimized to $0.05 \mathrm{eV}^{-1}$. For rutile (110) oxides, $\mathrm{MgO}$ (110), FCC (111) metals, and doped MoS2, a $(6,6,1),(4,4,1),(4,4,1)$, or $(2,2,1) \mathrm{k}$-point sampling was employed on a $2 \times 1,4 \times 2,3 \times 3$, or $3 \times 4$ expansion of the surface unit cell. Metal and oxide slabs were composed of four stoichiometric layers separated by 15 $\AA$ A vaccum; the lowest 2 layers were kept fixed to simulate the bulk. For doped $\mathrm{MoS}_{2}$ geometries, the sulfur atoms in bottom of the 2D network were fixed. For each active site motif and material category, a ClimbingImage Nudged Elastic Band (CI-NEB) ${ }^{22}$ calculation was performed to determine the location of the transition state (TS). Adsorption energies were referenced to gas-phase $\mathrm{O}_{2}, \mathrm{CH}_{4}$, and $\mathrm{H}_{2} \mathrm{O}$. 


\section{References}

1. Horn, R. \& Schlog1, R. Methane Activation by Heterogeneous Catalysis. Catalysis Letters 145, 23:39 (2014).

2. Labinger, J. A. \& Bercaw, J. E. Understanding and exploiting C-H bond activation. Nature 417, 507-14 (2002).

3. Zavyalova, U., Holena, M., Schlögl, R. \& Baerns, M. Statistical analysis of past catalytic data on oxidative methane coupling for new insights into the composition of highperformance catalysts. ChemCatChem 3, 1935-1947 (2011).

4. de Vekki, A. V. \& Marakaev, S. T. Catalytic partial oxidation of methane to formaldehyde. Russ. J. Appl. Chem. 82, 521-536 (2009).

5. Periana, R. a. Platinum Catalysts for the High-Yield Oxidation of Methane to a Methanol Derivative. Science (80-. ). 280, 560-564 (1998).

6. Woertink, J. S. et al. A [Cu2O $] 2+$ core in $\mathrm{Cu}-\mathrm{ZSM}-5$, the active site in the oxidation of methane to methanol. Proc. Natl. Acad. Sci. U. S. A. 106, 18908-18913 (2009).

7. Palkovits, R. et al. Development of molecular and solid catalysts for the direct lowtemperature oxidation of methane to methanol. ChemSusChem 3, 277-282 (2010).

8. Hammond, C., Conrad, S. \& Hermans, I. Oxidative methane upgrading. ChemSusChem 5, 1668-1686 (2012).

9. Kumar, G., Lau, S. L. J., Krcha, M. D. \& Janik, M. J. Correlation of Methane Activation and Oxide Catalyst Reducibility and Its Implications for Oxidative Coupling. ACS Catal. 1812-1821 (2016).

10. Zhao, Z.-J., Kulkarni, A., Vilella, L., Norskov, J. K. \& Studt, F. Theoretical insights into the selective oxidation of methane to methanol in copper-exchanged mordenite. ACS Catal. acscatal.6b00440 (2016).

11. Deshlahra, P. \& Iglesia, E. Reactivity and Selectivity Descriptors for the Activation of C$\mathrm{H}$ Bonds in Hydrocarbons and Oxygenates on Metal Oxides. J. Phys. Chem. C acs.jpcc.6b04604 (2016).

12. Latimer, A. A. et al. Understanding trends in $\mathrm{C}-\mathrm{H}$ bond activation in heterogeneous catalysis. Nat. Mater. 1, 1-6 (2016).

13. Wang, S. et al. Universal transition state scaling relations for (de)hydrogenation over transition metals. Phys. Chem. Chem. Phys. 13, 20760 (2011).

14. Tsai, C., Latimer, A. a., Yoo, J. S., Studt, F. \& Abild-Pedersen, F. Predicting PromoterInduced Bond Activation on Solid Catalysts Using Elementary Bond Orders. J. Phys. Chem. Lett. 6, 3670-3674 (2015).

15. Yoo, J. S., Khan, T. S., Abild-Pedersen, F., Nørskov, J. K. \& Studt, F. On the role of the surface oxygen species during $\mathrm{A}-\mathrm{H}(\mathrm{A}=\mathrm{C}, \mathrm{N}, \mathrm{O})$ bond activation: a density functional theory study. Chem. Commun. 51, 2621-2624 (2015).

16. Hibbitts, D. \& Neurock, M. Promotional effects of chemisorbed oxygen and hydroxide in the activation of C-H and O-H bonds over transition metal surfaces. Surf. Sci. 650, 210220 (2016).

17. Hereijgers, B. P. C. \& Weckhuysen, B. M. An Attempt to Selectively Oxidize Methane over Supported Gold Catalysts. Catal. Letters 141, 1429-1434 (2011).

18. Aljama, H., Yoo, S., Nørskov, J. K. \& Abild-pedersen, F. Methanol Partial Oxidation on Ag ( 111 ) from First Principles. 1-6 (2016).

19. Abild-Pedersen, F. et al. Scaling properties of adsorption energies for hydrogen- 
containing molecules on transition-metal surfaces. Phys. Rev. Lett. 99, 6105 (2007).

20. Chretien, S. \& Metiu, H. Acid - Base Interaction and Its Role in Alkane Dissociative Chemisorption on Oxide Surfaces Steeve Chre t. J. Phys. Chem. C 27336-27342 (2014).

21. Lopez, N. \& Garcia-Melchor, M. Dissociation on Metal Oxides : The example of CeO Homolytic Products from Heterolytic Paths in H 2 Dissociation on Metal Oxides : The example of CeO 2. J. Phys. Chem. C 10921-10926 (2014).

22. Henkelman, G., Uberuaga, B. P., Jónsson, H. \& Henkelman, G. A climbing image nudged elastic band method for finding saddle points and minimum energy paths A climbing image nudged elastic band method for finding saddle points and minimum energy paths. 9901, 1-5 (2011). 